\title{
Ankylostomidose, Ascaridose et Trichuriose associées dans une meute de chiens courants.
}

\author{
Trois ans d'essai de contrôle du parasitisme \\ par le Pyrantel pamoate. (1)
par J.-P. RAYNAUD *, J.-P. GONNEAUD ** et G. PLESSIS *** «Pfizer International », B.P. 42, F 37400 Amboise \\ * Station de Recherche et Développement vétérinaire et Nutrition animale \\ (Collaboration technique: G. William * et G. Brunault *)
}

\begin{abstract}
Résumé
Nous avons contrôlé pendant plus de 3 ans le parasitisme intestinal dans une meute de 50 chiens de race française (Poitevine Saintongeaise) courant régulièrement le chevreuil en une saison de chasse débutant le $1^{\text {er }}$ octobre et finissant le $1^{\text {er }}$ avril.

Ce parasitisme était constitué :

- d'Ankylostomidés (Uncinaria sp. représentant 90 à $95 \%$ des œufs éliminés, Ancylostoma sp. 5 à $10 \%$ );

- d'Ascaridés (Toxocara sp.);

- de Trichuridés (Trichuris sp.).

Nous avons utilisé un anthelminthique le Pyrantel sous forme de pamoate, sel peu soluble, lentement résorbable chez le chien, à la dose de $21 \mathrm{mg}$ par $\mathrm{kg}$ de poids vif (équivalent en Pyrantel base : $7,2 \mathrm{mg}$ ).

Cet anthelminthique est connu pour supprimer $100 \%$ des Ankylostomides et des Ascarides à partir d'une dose 3 fois moindre. Il n'est pas efficace sur Trichuris. Il est dépourvu de toxicité.

En ce qui concerne Trichuris le parasitisme jugé sur les coproscopies quantitatives a semblé se maintenir constant pen-

\footnotetext{
** 17, rue Sylvain-Fouassier, F. 53200 Château-Gontier.

*** 3, avenue Wilson, F. 79200 Parthenay.
}

(1) Pyrantel pamoate: Nemex, combantrin (N.D. Pfizer).
\end{abstract}


dant plus de 2 ans à un niveau non négligeable mais n'affectant pas la santé des animaux (une intervention systématique contre ce parasite pourrait être appliquée hors la saison de chasse avec certaines précautions nécessitées par le risque de toxicité des produits efficaces).

En ce qui concerne Ascaris (Toxocara) le traitement systématique des lices et des chiots avant le sevrage est pleinement efficace.

En ce qui concerne Uncinaria et Ancylostoma le parasitisme, une fois éliminé, redevient patent avant la saison de chasse, donc de surmenage. Il peut même devenir fort grave au point de compromettre les performances et la santé des animaux.

Les traitements au Pyrantel pamoate ont été parfaitement efficaces; malgré cela nous n'avons pas réussi à éliminer les parasites. Nous pensons que l'infestation s'entretient au chenil, les larves y trouvant des fissures ou recoins de la dalle cimentée, ou dans le terrain sec de l'aire d'exercice. La contamination pourrait aussi être extérieure au chenil (autres chiens ou renards).

Le programme de traitement de routine devrait être :

1) Si l'on pratique les contrôle coproscopiques: lorsque la moyenne globale des œufs dépasse 100 à 200 par gramme de matière fécale (intervention tactique).

2) Sans contrôle coproscopique :

- Il doit être réalisé lorsque l'ensemble des matières fécales est de consistance pâteuse ou liquide (seuil plus précoce que l'indication des performances cynégétiques ou l'observation de \& l'allant» à la course).

- En intervention stratégique :

a) En milieu ou fin de saison de chasse.

b) Une fois avant la saison de chasse (mois d'août par exemple).

\section{Summary}

We have controlled during more than three years intestinal parasitism in a pack of 50 dogs of a French race (Poitevine-Saintongeaise) regularly chasing roe deer during hunting seasons from the first of October to the first of April.

The parasitism was made up of:

- Hookworms (Uncinaria sp. representing 90 to $95 \%$ of the excreted eggs, Ancylostoma sp. 5 to $10 \%$ ).

- Ascarids (Toxocara sp.).

- Trichurids (Trichuris sp.).

We have used an anthelmintic Pyrantel as pamoate, salt of low solubility and of slow reabsorption in the dog, at a dose of $21 \mathrm{mg} / \mathrm{kg}$ live weight. (Pyrantel base equivalent : 7,2 mg/kg). 
This anthelmintic is known to remove $100 \%$ of Hookworms and Ascarids already at dosage of 3 times less. It is not effective on Trichuris. It is non-toxic.

Concerning Trichuris the parasitims judged by quantitative coproscopy seemed to remain constant during two years at a non-negligeable but not affecting the health of the animals level.

(A systematic intervention against the parasite could be applied out of the hunting season with certain precautions necessitated by the risk of toxicity of effective products).

Concerning Ascaris (Toxocara) systematic treatment of bitches and of pups before weaning is fully effective.

Concerning Uncinaria and Ancylostoma parasitism, once eliminated, returns surely before the hunting season, thus before hard work. It may even become grave enough to compromise the performance and health of the animals.

Treatments with Pyrantel pamoate have been perfectly effective, but in spite of this we have been unable to succeed in elimination of the parasites. We think that infestation occurs in the kennel, the larvae finding there cracks or corners in the cemented terrace or in the dry ground of the exercice area. Contamination could also be exterior to the kennel (other dogs or foxes).

The routine of treatment should be :

1) If coproscopic control is practised: when the average of eggs counts exceeds 100 to $200 / \mathrm{g}$ feces (tactical intervention).

2) Without coproscopic control :

- Must be done when the fecal consistency is pasty or liquid for all the animals (earlier threshold than an indication in cynegetic performances or the observation of «the running » during the chase).

- As strategic intervention:

a) In the middle or at the end of the hunting season.

b) Once before the hunting season (in August, for example).

L'ankylostomidose (parasitisme par Ancylostoma et/ou Uncinaria) est fréquente chez les chiens de chasse où elle est souvent désignée sous le nom d' "Anémie des Meutes» ou «Saignement de nez des chiens de meutes».

Dans la bibliographie anglo-saxonne, citons l'introduction d'un article récent de T. A. Miller (1971) «Par comparaison à son équivalent en médecine humaine l'ankylostomidose canine semble plutôt négligée sur le plan des recherches et des publications. On trouve des descriptions de la maladie dans la plupart des ouvrages d'helminthologie et parasitologie vétérinaire (Moning, 1947, Soulsby, 1965, Lapage, 1968, Georgi, 1969). L'uniformité des descriptions données est remarquable. Avec quelques exceptions mineures, les descriptions semblent avoir été recopiées consciencieusement pendant les der- 
nières 40 années. Ceci ne signifie pas que les expérimentations donnent des résultats uniformes mais plutôt qu'elles ne sont pas réalisées... »

Ce jugement est évidemment très sévère. En France, nous disposons de l'étude exhaustive des parasites et de l'affection chez le chien par J. EuzeBy 1963. Selon cet auteur \&l'Ankylostomose vraie est la plus souvent en cause dans ce syndrome, mais l'association Ankylostomose + Uncinariose et même Trichuriose est souvent aussi observée. Cette «anémie des meutes» est l'homologue de «l'anémie des mineurs » ankylostomose de l'homme due à Ankylostoma duodenale ».

Nous avons pour notre part étudié et suivi pendant plus de trois ans les parasites intestinaux dans une meute de 50 chiens de race Poitevine-Saintongeaise courant régulièrement le chevreuil. Ce travail a été détaillé pour une part dans la thèse de l'un d'entre-nous (G. Plessis, 1971).

\section{Le problème.}

Affections parasitaires intestinales et leur contrôle.

Avant notre première intervention, c'est-à-dire au cours de la saison de chasse octobre 1967 - Avril 1968, les animaux avaient présenté une affectation clinique diagnostiquée par l'un d'entre nous comme « Ankylostomidose » (c'est-à-dire Ankylostomose et/ ou Uncinariose). Ils avaient été traités avec succès au Tétrachloréthylène mais l'affection grave avait fortement perturbé la saison de chasse.

L'objectif des soigneurs était de maintenir un état de santé à un niveau maximum pendant la saison de chasse (soit du $1^{\text {er }}$ octobre au $1^{\text {er }}$ avril). Ils ne pouvaient et ne voulaient utiliser en traitement systématique des produits classiques ou nouveaux (Gibson T. E., 1965, Basseporte G., 1969) tels que Tétrachloréthylène, Thénium, Tétramisole ou Dichlorvos car en pleine saison de chasse ils ne voulaient prendre le risque de troubles ou baisse de performances dus à l'un de ces traitements. Comme nous le verrons plus loin, il nous faut considérer la meute comme une équipe de sportifs surentraînés faisant deux sorties par semaine, soit 30 à $50 \mathrm{~km}$ de course en terrain varié.

L'affection parasitaire semblait se développer en cours de saison de chasse jusqu'à atteindre un niveau dangereux en fin de saison (à partir du mois de mars) susceptible de la compromettre.

Il nous fallait donc utiliser un anthelminthique efficace bien sûr, mais aussi d'une parfaite innocuité pour traiter mâles et femelles en cours de saison de chasse. C'est ce qui nous a orientés sur le Pyrantel et son sel non résorbé dans le tube digestif, le pamoate. Plus précisément, le Pyrantel pamoate n'est pas résorbé dans l'intestin de l'homme tandis qu'il l'est, mais lentement dans celui du chien. Il est proposé aujourd'hui pour la thérapeutique humaine (4) ou celle du chien (5).

Nous joignons des éléments de bibliographie concernant le Pyrantel pamoate - son utilisation chez l'homme (1-2-4-5-7-8-10-15-17-18) et chez le chien (9-2؟) - ainsi que le Pyrantel chlorhydrate ou tartrate chez le chien (6-11-16).

Pyrantel pamoate: 4) Thérapeutique humaine: Combantrin (R). 5) Thérapeutique canine: Nemex (R.) : Noms déposés Pfizer. 


\section{Matériel et méthodes.}

\section{LES CHIENS.}

La meute «Rallye Anjou » fut créée à la fin du XIX ${ }^{e}$ siècle par le Vicomte Olivier de Rougé fondateur en outre de la race bovine Maine-Anjou.

Les chiens qui la composent sont de race Poitevine-Saintongeaise avec un rappel de sang anglais; leur couleur est tricolore. Depuis 1958, M. Gérard de Rougé assume la responsabilité des destinées du Rallye. La meute est installée depuis 1967 au Château de Bréon dans la commune de Daon (Mayenne).

M. Godivier, Bouton d'Equipage est le responsable de la meute. Un piqueux assure les soins et l'entretien des chiens. Il prend part à la chasse sous la direction du Maître d'Equipage.

La meute se compose de 50 chiens (photos 1). En dehors des périodes de mises bas, il y a 30 mâles, 10 femelles et 10 jeunes de moins d'un an. Le nombre des femelles est volontairement réduit pour éviter les perturbations créées par les chiennes lors de la chasse. La sélection s'opère sur la seule aptitude à la chasse.

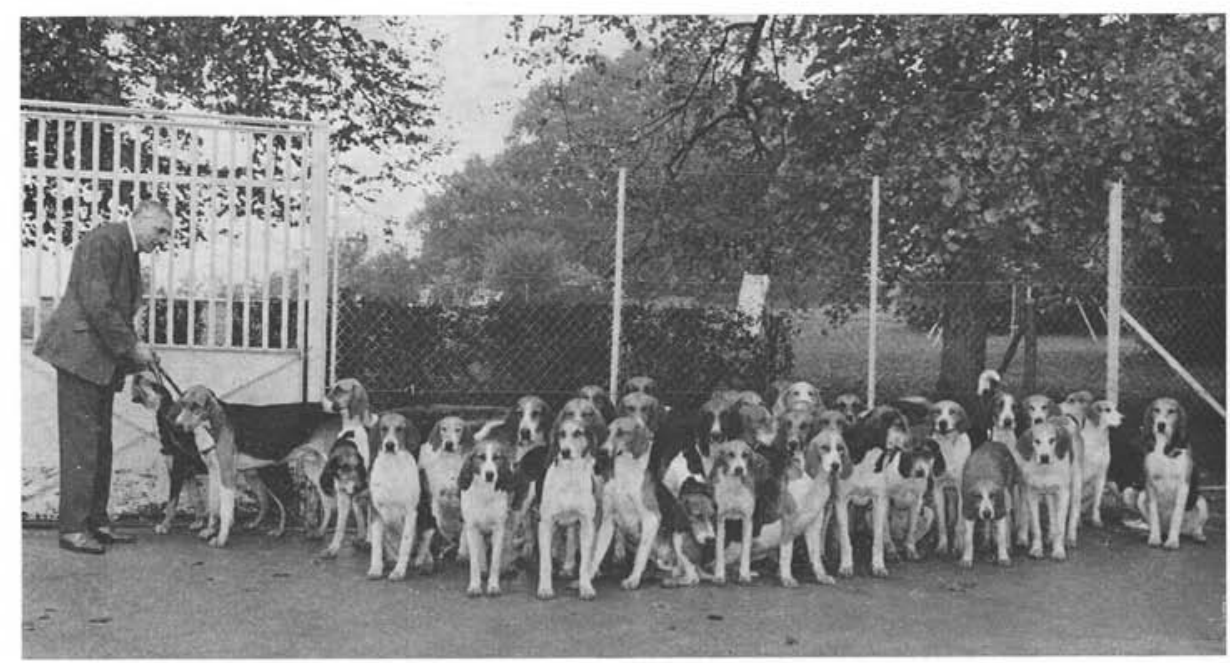

Pното 1. - La meute « Rallye Anjou» et M. Godivier, Bouton d'Equipage, sur l'aire de repos cimenté

La meute complète doit normalement courir exception faite des chiennes « en feu », en fin de gestation, ou allaitantes.

\section{LEUR NOURRITURE.}

Leur nourriture journalière comprend 80 kilos de viande, 15 kilos de farine d'orge. Deux fois par semaine, la farine d'orge est remplacée par le même poids de riz poli. La préparation de cette nourriture s'effectue dans un bâtiment préfabriqué adjacent au chenil. Les aliments sont distribués dans des bacs montés sur roulettes, après une cuisson lente de douze heures. 
L'eau est fournie sur l'aire de repos dans un bac fixe, de propreté convenable.

LE CHENIL.

Il comprend :

- Une aire de repos cimentée: niche commune cimentée avec litières de paille sur les caillebotis à $40 \mathrm{~cm}$ du sol. Cette litière est renouvelée deux fois par semaine ; la niche se prolonge d'une cour de $140 \mathrm{~m}^{2}$ ombragée par de hauts tilleuls.

- Une aire d'exercice de terre avec graviers, plantée de quelques pommiers, d'une superficie de $1000 \mathrm{~m}^{2}$ environ. Elle est fermée sur trois côtés par un mur de $2,50 \mathrm{~m}$ de haut et un grillage sur l'Ouest (photo 2 ).

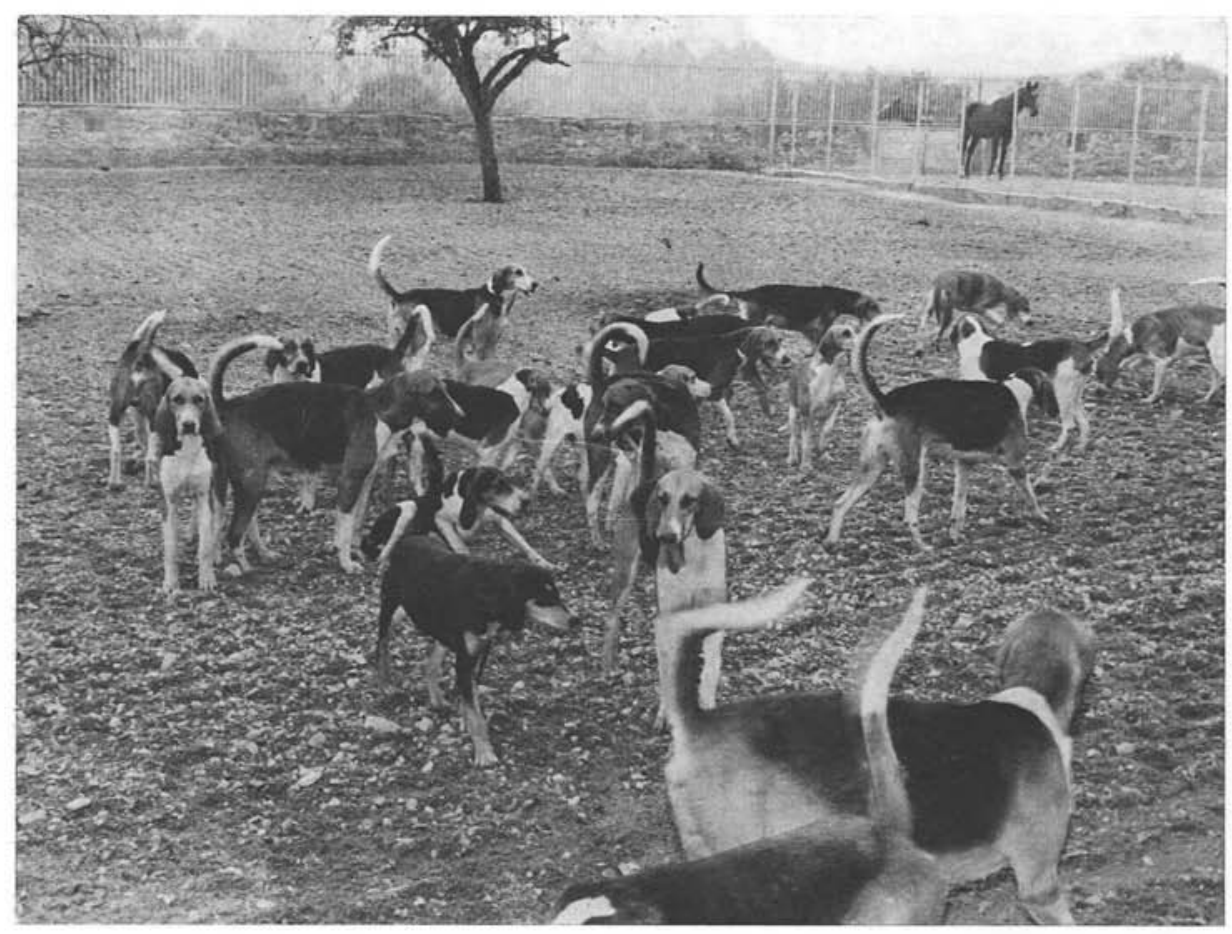

Рното 2. - Aire d'exercice

Pour les chiennes en chaleurs et les lices à la mise bas et en allaitement une niche d'isolement contiguë à la niche de repos avec une cour cimentée de $30 \mathrm{~m}^{2}$ environ.

\section{Entretien du chenil.}

La partie cimentée est débarassée tous les jours des déjections à l'aide d'un jet d'eau sous pression. L'eau est évacuée grâce à une légère pente et ne stagne pas.

La paille des litières est renouvelée fréquemment. 


\section{Prélèvement des matières fécales.}

\section{Leur utilisation au Laboratoire.}

Nous n'avons trouvé d'autre solution que le prélèvement tôt le matin des excréments pris séparément sur le sol, celui-ci étant encore plus soigneusement nettoyé la veille qu'à l'habitude.

Un certain nombre d'animaux peut être isolé à chaque série de prélèvements. Certains ont ainsi pu être suivis tout au long de l'expérimentation.

Les prélèvements sont mis dans des boîtes individuelles portant soit le nom de l'animal, soit un numéro mis au hasard. Les boîtes mises en caisse isotherme (type glacière de camping) avec des sachets de glace artificielle maintenant le froid sont expédiées en express au Laboratoire à Amboise.

La technique de coproscopie que nous pratiquons est celle publiée (J. P. Raynaud, 1970). Il s'agit d'une coproscopie quantitative en lame de Mac Master et examen complémentaire d'une lame de flottaison, le liquide de flottaison étant l'Iodo Mercurate de Potassium. Avec cette technique le seuil est à 0 . C'est-à-dire que lorsque nous portons 0 dans nos résultats cela signifie 0 /par gramme dans un prélèvement de 5 grammes de matières fécales. Il s'agit d'une technique dont nous avons démontré la sensibilité.

Au moment de l'analyse au laboratoire, la consistance des matières fécales est notée sous forme d'un Index des Diarrhées. Ces coefficients sont proches de ceux donnés par J. Euzeby (1958), consistance normale $=1$, pâteuse $=2$, semi liquide $=3$, diarrhéique $=4$. Mais contrairement à ses indications, « on apporte, si besoin est, les corrections nécessaires tenant compte de la consistance de matières fécales en utilisant ces coefficients », nous n'avons pas apporté de correction à nos résultats de numération car cela les aurait portés à des sommets peu vraisemblables. Cette correction peut cependant être toujours envisagée et exécutée $a$ posteriori.

\section{L'anthelminthique: Pyrantel Pamoate.}

Le pyrantel ou « Trans-1-méthyl-2 (thionylvinyl) 1, 4, 5, 6 tétrahydropyrimidine » a été décrit et expérimenté sous plusieurs formes pharmaceutiques (comprimés, granulés, poudre soluble ou insoluble, solution vraie, suspension etc...) le principe actif étant l'un des sels suivants : chlorhydrate, tartrate, ou pamoate. On a pu démontrer qu'à la même dose en «équivalent base » correspondait la même activité.

Cette équivalence s'établit suivant les masses moléculaires respectives: 7,2 mg de base $=8,3 \mathrm{mg}$ de chlorhydrate $=12,5 \mathrm{mg}$ de tartrate $=20,9 \mathrm{mg}$ de pamoate.

Chez le chien différents auteurs Howes H. L. et all. 1967, Cornwell R. L. et Jones R. M. 1968 ont démontré :

- La très grande efficacité du produit à dose très faible contre Ancylostoma caninum, Uncinaria stenocephala et Toxacara canis qui sont éliminés à 99-100\% par $2,5 \mathrm{mg}$ de tartrate de Pyrantel par $\mathrm{kg}$ de poids vif (équivalent à 4,2 $\mathrm{mg}$ de pamoate de Pyrantel par $\mathrm{kg}$ de pois vif). Ancylostoma caninum est ausi éliminé à $100 \%$ par $6 \mathrm{mg}$ de pamoate de Pyrantel par $\mathrm{kg}$ de poids vif. 
- L'absence d'activité du produit sur Trichuris vulpis (6) et divers Cestodes du chien (Taenia pisiformis et Dipylidium caninum).

Le Pyrantel pamoate a été choisi pour notre expérimentation car il est lentement résorbé chez le chien. Il ne peut gêner les animaux. C'est lui qui est utilisé pour l'homme.

Il a été administré le matin à jeun dans une capsule de gélatine à la dose de $20,9 \mathrm{mg}$ de pamoate de Pyrantel par $\mathrm{kg}$ de poids vif, (équivalent à $12,5 \mathrm{mg}$ en tartrate) et donc plus de trois fois la dose nécessaire à l'élimination de $100 \%$ des Ancylostoma suivant les résultats publiés.

\section{OBJECTIFS.}

Nous étions concernés par les parasites décelés par les coproscopies, par les index de diarrhées.

Le propriétaire et les piqueux étaient concernés par les performances de ces animaux de sport fortement sollicités en saison de chasse.

Le contrôle de leur dynamisme était un bon baromètre de leur état de santé, mais nous en examinerons les limites.

L'expérience nous a prouvé que le parasitisme intestinal se développait pendant la saison de chasse.

Notre objectif a été, pendant cette période de trois ans de vérifier si le traitement avec un produit très efficace à un maximum de parasitisme jugé par la coproscopie, permettrait de contrôler et supprimer l'infestation.

RÉsultats.

1) Nous avons porté dans le tableau I le résultat des coproscopies et index de diarrhées avec pour les premiers les valeurs moyennes sur une trentaine de prélèvements environ, les écarts types de la moyenne et les extrêmes des valeurs individuelles. Ces chiffres sont donnés pour les trois groupes de parasites rencontrés: Ancylostoma Uncinaria ou Trichuris ou Ascaris.

Pour les index de diarrhées nous avons donné les valeurs moyennes et individuelles extrêmes.

2) Nous avons figuré tous les résultats moyens des coproscopies en un diagramme qui porte :

- En abscisses le temps, chaque ligne verticale était le premier de chaque mois. Le contrôle expérimental a porté sur 40 mois.

— En ordonnées 3 tableaux superposés qui sont à la même échelle : racine cubique ainsi qu'indiqué par L. F. Taffs et al. (1969). Comme pour ces auteurs cette échelle nous permet de mieux visualiser les coproscopies moyennes, inférieures ou égales à 200 œufs par gramme (cas de 36 prélèvements sur 57 séries) et surtout inférieures à 50 œufs par gramme (cas de 25 prélèvements sur 57 séries

(6) Encore qu'un auteur J.-P. Dessors (1971) ait publié récemment des informations d'une activité importante du Pyrantel sur Trichuris vulpis lorsqu'il est utilisé en solution. 


\section{Tableau 1}

Résultats des coproscopies et index de diarrhées. Valeurs moyennes $\pm \mathrm{SX}$ et (extrêmes) Coproscopies: œufs par $\mathrm{g}$ de matières fécales. Index de diarrhées de 1 (normal) à 4 (diarrhéique). Entre parenthèses le nombre de prélèvements ?: une seule valeur positive : moyenne non valide

\begin{tabular}{|c|c|c|c|c|c|}
\hline \multirow{2}{*}{ Année } & \multirow{2}{*}{$\begin{array}{l}\text { Jour (Nombre } \\
\text { de prélèvements) }\end{array}$} & \multirow{2}{*}{$\begin{array}{c}\text { Index } \\
\text { Diarrhées }\end{array}$} & \multicolumn{3}{|c|}{ Coproscopies } \\
\hline & & & Ancylos-uncin. & Trichuris & Ascaris \\
\hline \multirow{5}{*}{$\begin{array}{r}1968 . \\
\text { T. }\end{array}$} & 3 juil. (31). & $\begin{array}{c}2 \\
\text { (tous 2) }\end{array}$ & $\begin{array}{l}1261 \pm 238 \\
(100-5750)\end{array}$ & $\begin{array}{c}23 \pm 13 \\
(0-400)\end{array}$ & $\begin{array}{c}58 \text { ? } \\
(1 \text { à } 1 \text { 800) }\end{array}$ \\
\hline & \multicolumn{2}{|c|}{ Traitement 8 juillet. } & & & \\
\hline & 26 juil. (37) & $\begin{array}{c}1,0 \\
\text { (tous 1) }\end{array}$ & $\begin{array}{l}1 \pm 1 \\
(0-25)\end{array}$ & $\begin{array}{l}12 \pm 3 \\
(0-50)\end{array}$ & $\begin{array}{l}3 \pm 2 \\
(0-50)\end{array}$ \\
\hline & 8 août (32). & $\begin{array}{l}1,2 \\
(1-2)\end{array}$ & $\begin{array}{c}21 \pm 3 \\
(0-50)\end{array}$ & $\begin{array}{l}5 \pm 2 \\
(0-25)\end{array}$ & 0 \\
\hline & 17 oct. (28). & $\begin{array}{l}1,5 \\
(1-2)\end{array}$ & $\begin{array}{c}67 \pm 19 \\
(0-400)\end{array}$ & $\begin{array}{l}13 \pm 6 \\
(0-100)\end{array}$ & 0 \\
\hline \multirow[t]{3}{*}{1969.} & 10 janv. (25). & $\begin{array}{c}1,0 \\
\text { (tous 1) } \\
\end{array}$ & $\begin{array}{c}65 \pm 21 \\
(0-500)\end{array}$ & $\begin{array}{c}52 \pm 25 \\
(0-600)\end{array}$ & $\left(\begin{array}{l}2 \\
(1 \text { à } \\
50)\end{array}\right.$ \\
\hline & 23 avr. (24). & $\begin{array}{c}2,0 \\
\text { (tous 2) }\end{array}$ & $\begin{array}{c}1977 \pm 405 \\
(50-7850)\end{array}$ & $\begin{array}{c}1108 \pm 381 \\
\quad(0-8000)\end{array}$ & $\begin{array}{c}69 \pm 36 \\
(0-600)\end{array}$ \\
\hline & 30 avril (26). & $\begin{array}{c}1,7 \\
(1-4)\end{array}$ & $\begin{array}{c}3805 \pm 949 \\
(30-16050)\end{array}$ & $\begin{array}{c}1413 \pm 428 \\
(50-10500)\end{array}$ & $\begin{array}{l}129 \pm 58 \\
(0-1100)\end{array}$ \\
\hline \multirow{4}{*}{$\mathrm{T} \ldots$} & \multicolumn{2}{|c|}{ Traitement 30 avril. } & & & \\
\hline & 9 mai (27). & $\begin{array}{c}1,0 \\
\text { (tous 1) }\end{array}$ & $\begin{array}{l}12 \pm 9 \\
(0-250)\end{array}$ & $\begin{array}{c}831 \pm 184 \\
(0-3950)\end{array}$ & 0 \\
\hline & 22 mai (25). & $\begin{array}{l}1,5 \\
(1-4)\end{array}$ & $\begin{array}{c}400 \pm 221 \\
(0-4750)\end{array}$ & $\begin{array}{c}2278 \pm 254 \\
(0-10000)\end{array}$ & 0 \\
\hline & 25 juin (28). & $\begin{array}{l}1,5 \\
(1-4)\end{array}$ & $\begin{array}{c}290 \pm 78 \\
(0-1350)\end{array}$ & $\begin{array}{c}597 \pm 245 \\
(0-6150)\end{array}$ & 0 \\
\hline \multirow[t]{4}{*}{$\mathrm{T} \ldots$} & \multicolumn{2}{|c|}{ Traitement 17 juillet. } & & & \\
\hline & 30 juil. (28). & $\begin{array}{c}1 \\
\text { (tous 1) } \\
\end{array}$ & 0 & $\begin{array}{c}1671 \pm 503 \\
(7-13500) \\
\end{array}$ & $\begin{array}{r}463 ? \\
1 \text { seul à } 12900 \\
\end{array}$ \\
\hline & 16 oct. (17). & $\begin{array}{c}1 \\
\text { (tous } 1 \text { ) } \\
\end{array}$ & $\begin{array}{l}32 \pm 17 \\
(0-250)\end{array}$ & $\begin{array}{c}1082 \pm 744 \\
(0-12750) \\
\end{array}$ & $\begin{array}{c}208 \pm 143 \\
(0-2250)\end{array}$ \\
\hline & 5 déc. (23). & $\begin{array}{c}1,7 \\
(1-3)\end{array}$ & $\begin{array}{c}100 \pm 26 \\
(0-500)\end{array}$ & $\begin{array}{c}487 \pm 138 \\
(0-2150)\end{array}$ & $\begin{array}{c}135 \pm 40 \\
(0-600)\end{array}$ \\
\hline 1970 & 6 fév. (25). & $\begin{array}{c}1,7 \\
(1-4)\end{array}$ & $\begin{array}{l}192 \pm 75 \\
(0-1900)\end{array}$ & $\begin{array}{c}1825 \pm 822 \\
(0-20150)\end{array}$ & $\begin{array}{c}6 ? \\
1-150\end{array}$ \\
\hline \multirow{3}{*}{$\mathrm{T} \ldots$} & 29 mai (28). & $\begin{array}{c}3,0 \\
(1-4)\end{array}$ & $\begin{array}{l}394 \pm 97 \\
(0-1750)\end{array}$ & $\begin{array}{l}814 \pm 327 \\
(0-4000)\end{array}$ & 0 \\
\hline & \multicolumn{2}{|c|}{ Traitement 4 juin. } & & & \\
\hline & 5 nov. (30). & $\begin{array}{c}1,2 \\
(1-4)\end{array}$ & $\begin{array}{c}75 \pm 25 \\
(0-700)\end{array}$ & $\begin{array}{c}530 \pm 234 \\
(0-6500)\end{array}$ & 0 \\
\hline 1971. & 12 fév. (30). & $\begin{array}{c}1,0 \\
(1-2)\end{array}$ & $\begin{array}{c}69 \pm 19 \\
(0-350)\end{array}$ & $\begin{array}{l}354 \pm 90 \\
(0-1800)\end{array}$ & 0 \\
\hline \multirow[t]{4}{*}{$\mathrm{T} \ldots$} & \multicolumn{2}{|c|}{ Traitement 25 février. } & & & \\
\hline & 9 mars (18). & $\begin{array}{c}1,0 \\
\text { (tous 1) }\end{array}$ & $\begin{array}{l}5 ? \\
(1 \text { à } 90)\end{array}$ & $\begin{array}{c}1493 \pm 938 \\
(0-16900)\end{array}$ & 0 \\
\hline & 5 avril (32). & $\begin{array}{c}1,0 \\
\text { (tous 1) }\end{array}$ & 0 & $\begin{array}{c}1224 \pm 432 \\
(0-12500)\end{array}$ & 0 \\
\hline & 22 sept. (29). & $\begin{array}{c}1,6 \\
(1-3)\end{array}$ & $\begin{array}{l}230 \pm 87 \\
(0-1900)\end{array}$ & $\begin{array}{c}3305 \pm 1092 \\
(0-24200) \\
\end{array}$ & 0 \\
\hline $\mathbf{T} \ldots$ & \multicolumn{2}{|c|}{ Traitement 22 sept. } & & & \\
\hline
\end{tabular}



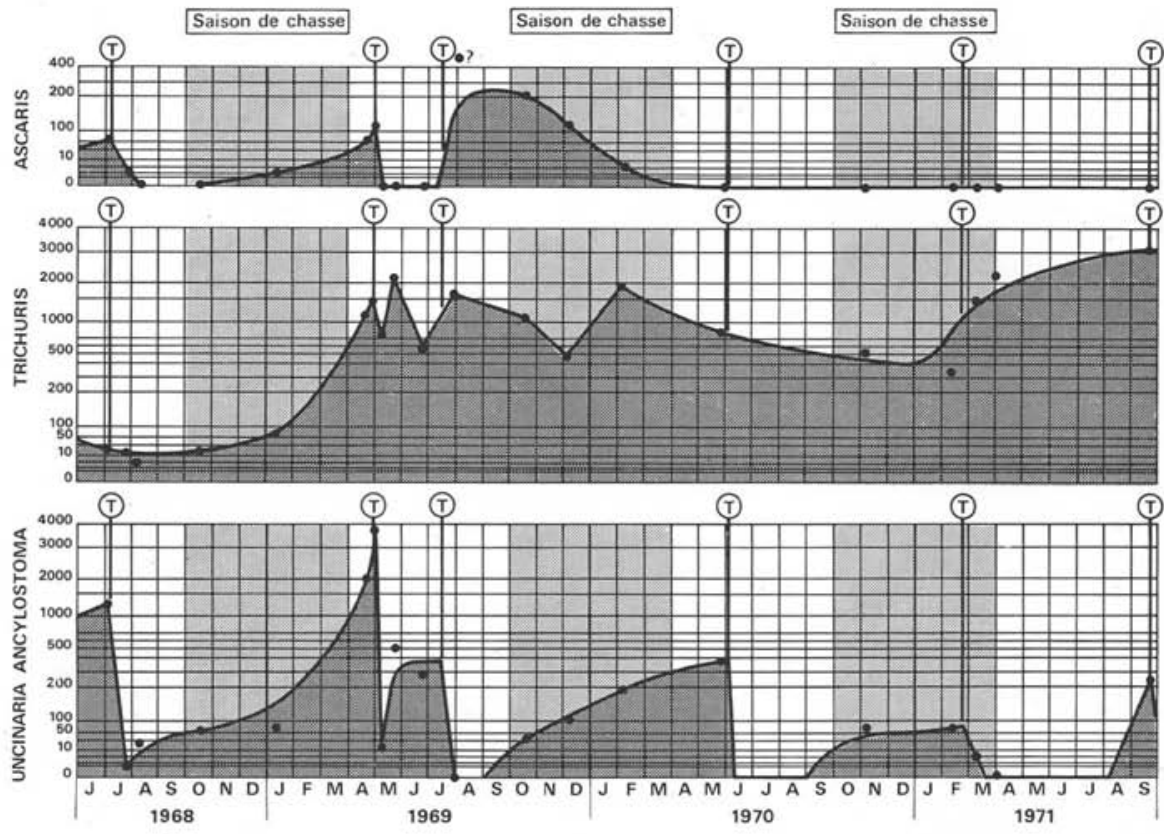

Diagramme: Résultats des coproscopies en œufs par gramme de matière fécale. Echelle $=$ racine cubique

examinées). Les tableaux correspondent aux groupes parasitaires rencontrés : Uncinaria, Ancylostoma, Trichuris ou Ascaris.

Sur ce diagramme les saisons de chasse (du $1^{\text {er }}$ octobre au $1^{\text {er }}$ avril) ont été soulignées en grisé. Les traitements systématiques ont aussi été indiqués par une verticale avec $\mathrm{T}$.

Nous n'avons pas figuré les traitements individuels faits en fin 1969 début 1970 sur les lices allaitantes et leurs chiots traités pour les débarrasser des ascaris.

3) Dans un tableau II nous avons porté quelques valeurs individuelles extrêmes pour les paramètres Index des diarrhées/Parasitisme ; il apparaît que certains animaux avec des matières fécales normales ont un nombre d'œufs éliminés très important alors que réciproquement d'autres avec des matières fécales semi-liquides ou diarrhéiques n'ont pas de parasitisme patent.

$\mathrm{Si}$ « l'index des diarrhées » n'a — ou n'aurait — pas de valeur à l'échelle de l'individu, par contre globalement on peut remarquer que les moyennes égales ou supérieures à 2,0 étaient :

— au 3 juillet 1968, date d'intervention justifiée ;

— au 23 avril 1969, date d'intervention justifiée ;

- au 30 avril 1969, date d'intervention nécessaire ;

- au 29 mai 1970, date d'intervention justifiée. 
Tableau 2

Relation. Index des diarrhées. Parasitisme (coproscopies individuelles en œufs par gramme)

\begin{tabular}{|c|c|c|c|c|c|}
\hline \multirow{2}{*}{ Prélèvement du } & \multirow{2}{*}{ Nom ou $n^{\circ}$} & \multirow{2}{*}{ I-D } & \multicolumn{3}{|c|}{ Coproscopies } \\
\hline & & & Unc.-Anc. & Tric. & Asc. \\
\hline 3 juil. $68 \ldots \ldots \ldots \ldots$ & $\begin{array}{l}\text { Paname } \\
\mathrm{n}^{\circ} 25\end{array}$ & $\begin{array}{l}2 \\
2\end{array}$ & $\begin{array}{r}100 \\
5750\end{array}$ & $\begin{array}{l}0 \\
0\end{array}$ & $\begin{array}{l}0 \\
0\end{array}$ \\
\hline 17 oct. $68 \ldots \ldots \ldots \ldots$ & $\begin{array}{ll}\mathrm{n}^{\circ} & 1 \\
\mathrm{n}^{\circ} & 23\end{array}$ & $\begin{array}{l}2 \\
1\end{array}$ & $\begin{array}{r}0 \\
300\end{array}$ & $\begin{array}{r}0 \\
50\end{array}$ & $\begin{array}{l}0 \\
0\end{array}$ \\
\hline 23 avr. $69 \ldots \ldots \ldots \ldots$ & $\begin{array}{l}\mathrm{n}^{\circ} 2 \\
\text { Pistache }\end{array}$ & $\begin{array}{l}2 \\
2\end{array}$ & $\begin{array}{r}250 \\
7850\end{array}$ & $\begin{array}{r}0 \\
2950\end{array}$ & $\begin{array}{l}0 \\
0\end{array}$ \\
\hline 31 avr. $69 \ldots \ldots \ldots$ & $\begin{array}{ll}\mathrm{n}^{\circ} & 22 \\
\mathrm{n}^{\circ} & 4\end{array}$ & $\begin{array}{l}1 \\
4\end{array}$ & $\begin{array}{r}16050 \\
350\end{array}$ & $\begin{array}{l}750 \\
100\end{array}$ & $\begin{array}{l}0 \\
0\end{array}$ \\
\hline 22 mai $69 \ldots$ & $\begin{array}{l}\mathrm{n}^{\circ} 19 \\
\text { Chienne }\end{array}$ & $\begin{array}{l}1 \\
4\end{array}$ & $\begin{array}{r}4750 \\
45\end{array}$ & $\begin{array}{r}4000 \\
0\end{array}$ & $\begin{array}{l}0 \\
0\end{array}$ \\
\hline
\end{tabular}

On peut donc attribuer une VALEUR INDICATIVE à la nature des matières fécales ; lorsque l'ensemble de la meute donne des matières pâteuses ou liquides, le traitement général peut être indiqué.

Par contre un examen de cette consistance à l'échelle de l'individu pourrait ne pas avoir de valeur indicative pour cet individu.

4) Dans le tableau III nous avons examiné le parasitisme des jeunes chiots en allaitement ou juste sevrés, des jeunes chiens sevrés et de l'ensemble de la meute.

Tableau 3

Parasites des chiots - jeunes chiens et adultes

\begin{tabular}{|c|c|c|c|c|c|}
\hline \multirow{2}{*}{ Année } & \multirow{2}{*}{$\begin{array}{l}\text { Jour de prélèv. } \\
\text { et groupes } \\
\text { (avec nombre } \\
\text { d'animaux) }\end{array}$} & \multirow{2}{*}{ Index } & \multicolumn{3}{|c|}{ Coproscopies } \\
\hline & & & $\begin{array}{l}\text { Ancyl- } \\
\text { uncin. }\end{array}$ & Tric. & Asc. \\
\hline \multirow[t]{2}{*}{$1969 \ldots$} & $\begin{array}{l}5 \text { décembre: } \\
\text { adultes (23) }\end{array}$ & $\begin{array}{c}1,7 \\
(1-3)\end{array}$ & $\begin{array}{c}100 \pm 26 \\
(0-500)\end{array}$ & $\begin{array}{c}487 \pm 138 \\
(0-2150)\end{array}$ & $\begin{array}{c}135 \pm 40 \\
(0-600)\end{array}$ \\
\hline & chiots (6) & $\begin{array}{c}1,2 \\
(1-2)\end{array}$ & $\begin{array}{c}233 \pm 42 \\
(0-600)\end{array}$ & $\begin{array}{c}1267 \pm 352 \\
(150-2750)\end{array}$ & $\begin{array}{l}430 \pm 112 \\
(100-1550)\end{array}$ \\
\hline \multirow[t]{2}{*}{$1970 \ldots$} & $\begin{array}{l}29 \text { mai : } \\
\text { adultes (28) }\end{array}$ & $\begin{array}{c}3,0 \\
(1-4)\end{array}$ & $\begin{array}{r}394 \pm 97 \\
(0-1750)\end{array}$ & $\begin{array}{c}814 \pm 327 \\
(0-4000)\end{array}$ & 0 \\
\hline & $\begin{array}{l}\text { jeunes chiens } \\
\text { (7) }\end{array}$ & $\begin{array}{c}3,4 \\
(1-4)\end{array}$ & $\begin{array}{l}360 \pm 78 \\
(60-1100)\end{array}$ & $\begin{array}{c}362 \pm 128 \\
(0-2000)\end{array}$ & 0 \\
\hline
\end{tabular}


Qualitativement et quantitativement le parasitisme est le même ; on peut donc en edédúíre que c'est bien au chenil avec leurs mères d'abord, avec Ia meute ensuite que les jeunes s'infestent.

5) Dans le tableau IV nous avons examiné l'hypothèse de la simultanéité apparente ou de l'exolusion apparente des infestations pour Uncinaria - Ancylostoma et Trichuris ou Ascaris.

Les exemples choisis sont loin des périodes de traitement et lorsque l'infestation à Trichuris est installée à un niveau qu'elle gardera d'ailleurs pendant le restant de Ia période de contrôle.

\section{Tableau 4}

'Simultanéité (S) ou exclusion (E) apparente des infestations à Ankylostomidés et Trichuris (coproscopies individuelles en æufs par gramme)

\begin{tabular}{|c|c|c|c|c|c|c|}
\hline \multirow[b]{2}{*}{ Prélèvement du } & \multirow{2}{*}{\multicolumn{2}{|c|}{ Nom ou $\mathrm{n}^{\circ}$}} & \multirow{2}{*}{ I-D } & \multicolumn{3}{|c|}{ Coproscopies } \\
\hline & & & & $\begin{array}{l}\text { Unc.- } \\
\text { Anc. }\end{array}$ & Tric. & Ase. \\
\hline 23 avr. $1969 \ldots \ldots$ & $\begin{array}{l}\text { Pistache } \\
\mathrm{n}^{\circ} 18\end{array}$ & $\begin{array}{l}\text { (S) } \\
\text { (S) }\end{array}$ & $\begin{array}{l}2 \\
2\end{array}$ & $\begin{array}{l}7850 \\
6000\end{array}$ & $\begin{array}{l}2950 \\
1100\end{array}$ & $\begin{array}{l}0 \\
0\end{array}$ \\
\hline 31 avr. $1969 \ldots$. & $\begin{array}{l}\text { Palatin } \\
\text { Périgord } \\
\mathrm{n}^{\circ} 15 \\
\text { Orient }\end{array}$ & $\begin{array}{l}\text { (S) } \\
\text { (S) } \\
\text { (S) } \\
\text { (E) }\end{array}$ & $\begin{array}{l}1 \\
1 \\
1 \\
4\end{array}$ & $\begin{array}{r}8550 \\
13450 \\
2300 \\
2200\end{array}$ & $\begin{array}{r}10500 \\
3850 \\
2900 \\
50\end{array}$ & $\begin{array}{c}0 \\
0 \\
1000 \\
0\end{array}$ \\
\hline 25 juin $1969 \ldots$ & $\begin{array}{ll}\mathrm{n}^{\circ} & 20 \\
\mathrm{n}^{\circ} & 12 \\
\text { Pernod }\end{array}$ & $\begin{array}{l}\text { (S) } \\
\text { (E) } \\
(\mathrm{E} ?)\end{array}$ & $\begin{array}{l}4 \\
1 \\
1\end{array}$ & $\begin{array}{r}1300 \\
100 \\
650\end{array}$ & $\begin{array}{ll}3 & 150 \\
2 & 000 \\
6 & 150\end{array}$ & $\begin{array}{l}0 \\
0 \\
0\end{array}$ \\
\hline 6 fév. $1970 \ldots \ldots$ & $\begin{array}{ll}\mathrm{n}^{\circ} & 19 \\
\mathrm{n}^{\circ} & 7 \\
\mathrm{n}^{\circ} & 18 \\
\mathrm{n}^{\circ} & 15 \\
\mathrm{n}^{\circ} & 9\end{array}$ & $\begin{array}{l}\text { (S) } \\
\text { (S) } \\
\text { (E) } \\
\text { (E) } \\
\text { (E) }\end{array}$ & $\begin{array}{l}1 \\
1 \\
1 \\
3 \\
2\end{array}$ & $\begin{array}{r}1900 \\
150 \\
0 \\
0 \\
0\end{array}$ & $\begin{array}{r}2800 \\
450 \\
20150 \\
1200 \\
850\end{array}$ & $\begin{array}{l}0 \\
0 \\
0 \\
0 \\
0\end{array}$ \\
\hline 29 mai $1970 \ldots$. & $\begin{array}{l}\text { Quichote } \\
\text { Orange } \\
\text { Madrigal } \\
\text { Orient }\end{array}$ & $\begin{array}{l}\text { (S) } \\
\text { (S) } \\
\text { (S) } \\
\text { (E) }\end{array}$ & $\begin{array}{l}3 \\
2 \\
3 \\
2\end{array}$ & $\begin{array}{rr}1 & 050 \\
1 & 750 \\
1 & 100 \\
& 0\end{array}$ & $\begin{array}{ll}4 & 000 \\
8 & 000 \\
3 & 450 \\
1 & 200\end{array}$ & $\begin{array}{l}0 \\
0 \\
0 \\
0\end{array}$ \\
\hline 5 nov. $1970 \ldots$ & $\begin{array}{ll}\mathrm{n}^{\circ} & 2 \\
\mathrm{n}^{\circ} & 15 \\
\mathrm{n}^{\circ} & 19\end{array}$ & $\begin{array}{l}\text { (S) } \\
\text { (E) } \\
\text { (E) }\end{array}$ & $\begin{array}{l}1 \\
1 \\
1\end{array}$ & $\begin{array}{r}700 \\
0 \\
0\end{array}$ & $\begin{array}{r}1750 \\
6500 \\
900\end{array}$ & $\begin{array}{l}0 \\
0 \\
0\end{array}$ \\
\hline 22 sept. $1971 \ldots$. & $\begin{array}{l}\text { Tartarin } \\
\text { Titus } \\
\mathrm{n}^{\circ} 2 \\
\text { Uranium } \\
\mathrm{n}^{\circ} 7 \\
\mathrm{n}^{\circ} 19\end{array}$ & $\begin{array}{l}\text { (S) } \\
\text { (S) } \\
\text { (S) } \\
\text { (E) } \\
\text { (E) } \\
\text { (E) }\end{array}$ & $\begin{array}{l}1 \\
1 \\
1 \\
1 \\
2 \\
2\end{array}$ & $\begin{array}{r}150 \\
350 \\
1900 \\
0 \\
0 \\
0\end{array}$ & $\begin{array}{r}150 \\
1000 \\
300 \\
12600 \\
900 \\
12550\end{array}$ & $\begin{array}{l}0 \\
0 \\
0 \\
0 \\
0 \\
0\end{array}$ \\
\hline
\end{tabular}


Il apparaît que confirmant les indications de J. Euzeby (1963) Uncinaria - Ancylostoma et Trichuris sont simultanément présents avec des éliminations d'œufs par gramme de matières fécales parfois fort élevées (respectivement 8550 et 10500 ou 13450 et 3850 ).

6) Uncinaria - Ancylostoma.

Le diagnostic des genres Uncinaria et Ancylostoma sur l'apparence des œufs est facile.

Nous l'avons fait et avons constaté que sur la durée de la période expérimentale le rapport des infestations semblait constant :

$$
\begin{aligned}
& \text { 90-95\% Uncinaria } \\
& \text { 5-10\% Ancylostoma }
\end{aligned}
$$

généralement associés.

7) Trichuris (Trichuris vulpis).

Les Trichuris étaient présents à un niveau très faible (23 œufs par g) lors de notre première intervention.

Il n'y eut pas de changement après le premier traitement et ce pendant 7 mois après ce traitement.

Au moment de notre $2^{\circ}$ intervention, 10 mois après la première, les Trichuris étaient éliminés régulièrement à une moyenne de 1000 œufs par gramme (extrêmes des moyennes 487 à 3 305) sans fluctuation importante ni relation avec la saison de chasse.

Nous avons confirmé l'inefficacité du Pyrantel pamoate sur Trichuris vulpis (au moins à cette dose et avec cette forme d'administration $=21 \mathrm{mg}$ de Pyrantel pamoate par $\mathrm{kg}$ de poids vif en une capsule de gélatine administrée à jeun) mais confirmé aussi que cette infestation régulière $=1000$ œufs environ par gramme de matière fécale n'avait aucun retentissement sur la santé des animaux quel que soit leur âge (dans le tableau $\mathrm{n}^{\circ} 3,6$ chiots éliminaient au sevrage 1267 œufs/g en moyenne).

Suivant J. Euzeby (1961) « on peut envisager une infestation maladie à partir de 6000 œufs par gramme de fécès », ce chiffre a été rarement rencontré dans cette meute mais si l'on examine le tableau IV on constate que quelques animaux en éliminaient un nombre supérieur sans aucun symptôme clinique. Ainsi :

- le 25 juin 1969 (Pernod = 6150 œufs/g);

- le 6 février 1970 (n $18=20150$ œufs/g);

- le 29 mai 1970 (Orange $=8000$ œufs/g);

- le 22 septembre 1971 (Uranium $=12600, \mathrm{n}^{\circ} 19=12550$ ).

Sur les animaux que nous avons contrôlés, un nombre considérable d'œufs de Trichures allant jusqu'à 10000 et même 20000 a pu être rencontré sans anomalie chez le chien porteur.

8) Ascaris (Toxocara canis).

Etaient présents dans la meute lors de notre première intervention, très irrégulièrement ( 1 animal seulement sur 30 environ).

Le 23 avril 1969, 5 chiens sur 24 étaient porteurs. 
Le 30 avril 1969, 7 chiens sur 26 étaient porteurs.

Le traitement fait ce jour les fit disparaître.

Les Ascaris réapparurent le 30 juillet 1969: un chien seulement éliminait 12900 œufs par gramme de matières fécales.

Ils furent éliminés à la suite de traitements individuels des lices et chiots au sevrage.

Depuis le $1^{\text {er }}$ juin 1970, ils ne sont plus décelés dans la meute.

9) Périodicité du parasitisme intestinal.

Nous avons vu :

- Que le parasitisme par Toxocara avait été facilement éliminé par les trois traitements systématiques de la meute et des traitements individuels des lices. Nous ne pouvons donc parler de périodicité.

- Que le parasitisme par Trichuris au contraire s'est généralisé et installé dans la meute à un niveau élevé. Nous n'avons pu lui attribuer une symptomatologie même fruste puisque ce parasitisme, jugé par les coproscopies quantitatives, était à peu près constant pendant plus de 2 ans.

Il y a donc pour Trichuris, absence complète de périodicité de l'infestation.

- Que le parasitisme par Uncinaria - Ancylostoma pouvait être directement ou indirectement attribué à la chasse ;

a) Il s'est trouvé pour notre première intervention supprimé ou neutralisé en période hors chasse (mais l'affection première avait éclaté en saison de chasse précédente).

b) Au cours de la seconde saison de chasse (1968-1969) ce parasitisme s'est développé lentement puis en courbe parabolique à la fin de cette saison.

Notre traitement du 31 avril 1969 était trop tardif ; il a dû être suivi d'un second traitement le 17 juillet qui a été très efficace puisque les Ankylostomidés avaient disparu.

C'est à la fin de cette saison que les animaux ont eux des performances médiocres enregistrées dans le «Livre du Rallye ».

c) Au cours de la troisième saison de chasse (1969-1970) le parasitisme s'est développé lentement, régulièrement, progressivement.

Aucune anomalie sur le plan des performances n'a été signalée ; en période hors chasse les animaux ont été traités le 4 juin sans que le traitement ait été jugé nécessaire par les soigneurs.

d) En quatrième saison de chasse (1970-1971) l'Ankylostomidose est réapparue ; elle s'est développée comme précédemment mais a été stoppée par un traitement précoce le 25 février.

e) La veille de la saison de chasse 1970-1971 ( $5^{\circ}$ saison) le parasitisme était revenu; il a été stoppé immédiatement par un traitement fait le 22 septembre. 


\begin{tabular}{|c|c|c|c|c|c|c|c|}
\hline \multirow{2}{*}{ 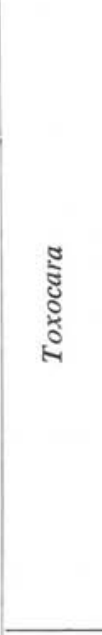 } & \multirow{2}{*}{\multicolumn{2}{|c|}{ 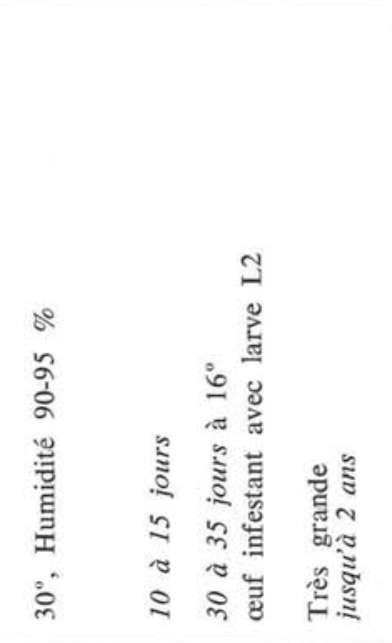 }} & 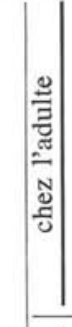 & \multicolumn{3}{|c|}{ 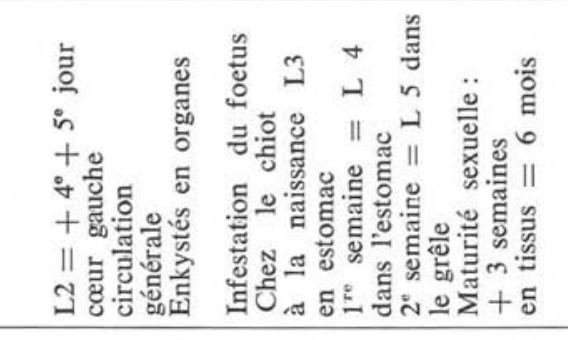 } & 1 \\
\hline & & & 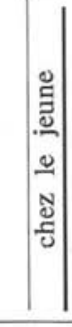 & 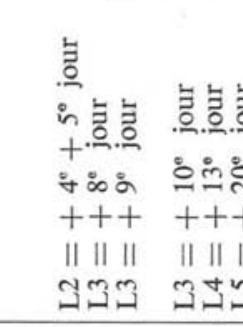 & & 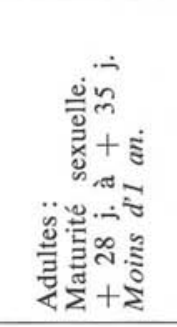 & '̃ \\
\hline 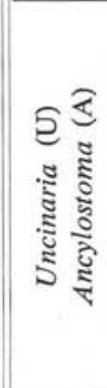 & 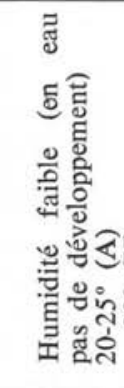 & 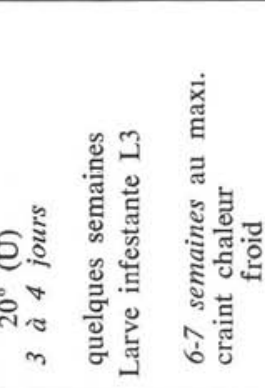 & 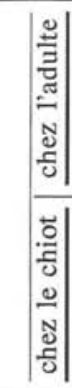 & $\begin{array}{l}7 \\
0\end{array}$ & 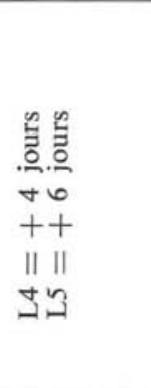 & 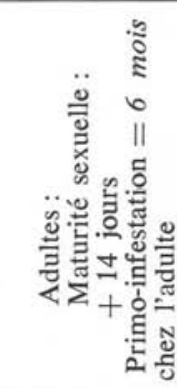 & 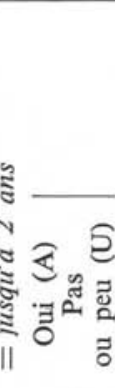 \\
\hline 玅 & 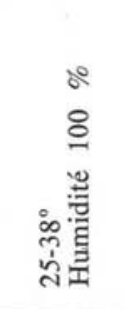 & 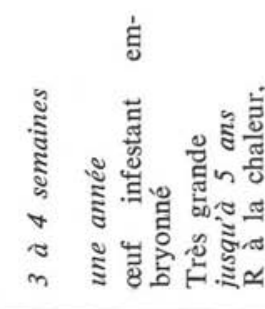 & & & 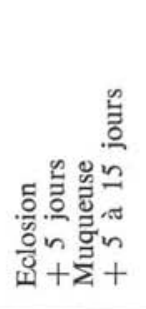 & 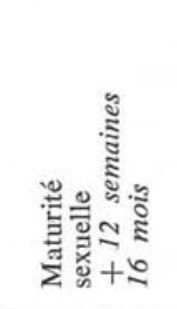 & 气̆ \\
\hline & 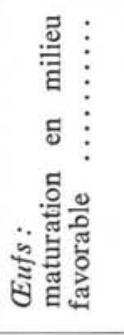 & 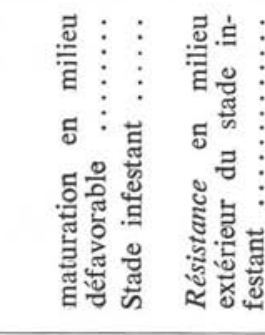 & 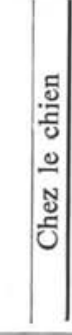 & 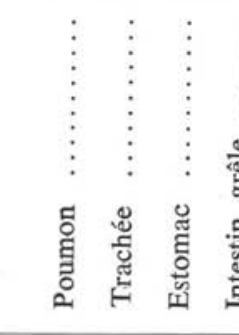 & 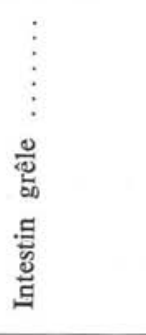 & 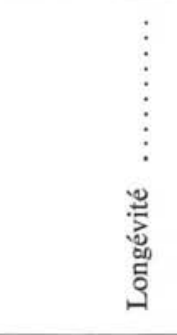 & 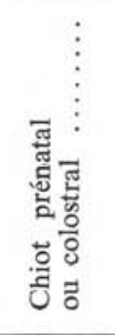 \\
\hline
\end{tabular}


On peut donc en conclure que le Pyrantel pamoate a régulièrement permis de supprimer le parasitisme par Uncinaria - Ancylostoma puisque les coproscopies étaient nulles après chaque intervention.

Il n’a pas réussi, malgré sa grande efficacité à éliminer le parasitisme dans la meute puisque celui-ci est réapparu à chaque saison de chasse. C'est ce point à la fois intéressant et important que nous allons examiner dans la discussion.

Discussion.

Nous allons essayer d'examiner les raisons pour lesquelles l'utilisation d'un anthelminthique efficace à $100 \%$ (dès la dose $1 / 3$ de celle que nous avons administrée) n'a pu supprimer le parasitisme par Uncinaria et Ancylostoma.

Pour cela considérons les éléments connus de la biologie des trois groupes de parasites que nou avons rencontrés (Tableau V). Suivant T. A. Miller 1971, E. J. L. Soulsby 1965, J. Euzeby 1961-1963, nous en extrayons :

\section{1) Pour Trichuris vulpis.}

Parasite a) dont le stade infestant est très résistant, la maturation depuis l'œuf fraîchement éliminé étant longue dans les conditions favorables (3 à 4 semaines) et pouvant être très longue dans des conditions défavorables.

Parasite $b$ ) dont la longévité est grande.

On comprend donc qu'un parasitisme installé dans une meute puisse s'entretenir régulièrement si l'anthelminthique utilisé n'a pas d'activité spécifique ce qui est le cas du Pyrantel pamoate.

2) Pour Toxocara canis.

L'œuf infestant est très résistant.

Mais l'infestation de l'adulte « est abortive et conduit le parasite à une impasse » (J. Euzeby, 1963).

Elle ne peut évoluer que dans le fœtus d'une femelle gestante. On comprend que le traitement individuel systématique des lices et des chiots sevrés, ait éliminé le parasite, le Pyrantel pamoate étant d'une grande efficacité contre les Ascaridés en général et Toxocara en particulier.

\section{3) Pour Uncinaria - Ancylostoma.}

On ne peut comprendre que le Pyrantel pamoate efficace à $100 \%$ contre ces parasites n'ait pu les éliminer complètement.

En effet, on remarque dans le tableau V, comme dans le diagramme, que sauf en 1968-1969 où le traitement était trop tardif :

- Il était correctement entrepris lorsque le parasitisme était faible en 1969-1970 et $1970-1971$.

- Dans ces deux derniers cas il était efficace puisqu'il a annulé les œufs dans les matières fécales.

- Mais il n'a pas empêché qu'un peu avant le début de la saison de chasse suivante les chiens ne recommencent à éliminer les œufs d'Uncinaria - Ancylostoma. 
Nous avons établi une corrélation nette entre le développement du parasitisme et le déroulement de la saison de chasse.

- L'hypothèse selon laquelle sur des animaux porteurs (mal guéris) un parasitisme latent est stimulé à la saison de chasse, n'est pas à retenir.

En effet, les œufs réapparaissent dans les matières fécales un mois environ avant le début de la chasse, à une époque où ils étaient correctement reposés.

- On peut admettre que l'infestation se fait au chenil. Elle peut être envisagée à partir d'animaux porteurs.

(Pour les résultats de 1971 on peut remarquer que le traitement du 25 février était très efficace puisque tous les prélèvements sauf un étaient nuls le 9 mars et tous étaient nuls le 5 avril. Pour les résultats de 1969 , le traitement du 17 juillet était très efficace puisque tous les prélèvements étaient nuls le 30 juillet).

Par contre, cette infestation peut très bien se faire après le traitement à partir des matières fécales contenant des œufs. Les larves résistent bien en milieu sec, et on peut toujours trouver des fissures ou recoins de la dalle cimentée où elles pourraient se nicher.

- Enfin on peut aussi penser à une contamination extérieure au chenil à partir du mois d'août, au cours de longues promenades faites pour entretenir leur condition physique; les sources de parasite possibles étant les seuls canidés pourraient être d'autres chiens (de chasse, domestiqués ou errants) ou même des renards nombreux dans la région.

\section{Conclusion.}

Nous avons confirmé que le traitement en cours de saison de chasse avec le Pyrantel pamoate ne présentait aucun inconvénient ; non plus que l'administration du produit aux chiennes gestantes ou allaitantes ou aux chiots.

Il nous paraît tout-à-fait indiqué en routine d'adopter l'un des programmes suivants pour la lutte contre Uncinaria - Ancylostoma.

1) Lorsqu'on peut faire un contrôle coproscopique, intervention tactique lorsque la moyenne globale des œufs dépasse 100 à 200 par $\mathrm{g}$ de matière fécale.

2) Sans contrôle coproscopique, lorsque l'ensemble des matières fécales est de consistance pâteuse ou liquide. Ce seuil est une indication de meilleure valeur plus précoce en tout cas que l'examen des performances cynégétiques.

En intervention stratégique :

a) Nécessairement une fois avant la saison de chasse (au mois d'août par exemple).

b) En milieu ou fin de saison de chasse.

En cas d'infestation importante par Trichuris un traitement spécifique pourra être appliqué hors la saison de chasse avec le «Whipcide» ou «Phthalofyne ou le 
Dichlorvos, en prenant les précautions recommandées pour éviter les risques de toxicité (administration de la dose juste nécessaire, repos, etc.).

En cas d'infestation par Ascaris (Toxocara) le traitement systématique individuel des lices et des chiots avant le sevrage avec le Pyrantel pamoate a montré sa valeur.

On devra appliquer les règles prophylactiques reconnues, en particulier le maintien d'une propreté rigoureuse du chenil; éventuellement on devra décider de cimenter entièrement les aires d'exercice. Lors de risque de transmission plus important (nous l'avons vu en cours de saison de chasse) on pourra être amené à pulvériser du borate de soude sur toutes les surfaces non cimentées.

\section{Bibliographie}

1. Amato Neto (V.), Lévy (G. C.) and Campos (L. L.), 1970. - (Findings in the antihelmintic activity of pyrantel pamoate I-Treatment of ascariasis). Rev. Inst. Med. Trop., Sao-Paulo, 12: 207-210.

2. Alexander (F.), Drykoningen (G.), Flahaut (J.) and Raveschot (H.), 1970. - VergelijKende studie over de therapeutische doeltreffendheid en tolerantie van het pyrvinium-pamoaat en het pyrantel pamoaat, angaande de besmetting met Enterobius vermicularis. Tschr. v. Geneesk $26^{\mathrm{e}}$ jg., $n^{\circ} 6$.

3. BASSEPorte (G.), 1969. - Médicaments spécifiques actuels contre les nématodes de l'intestin des carnivores domestiques. Etude comparative. Thèse Doctorat Vétérinaire. Faculté de Médecine de Toulouse.

4. Bell (W. J.) and Gould (G. C.), 1971. - Preliminary report on pyrantel pamoate in the treatment of human hookworm infection. East Africain Med. J., 48: 143-151.

5. Bell (W. J.) and NASsIF (S.), 1971. - Comparison of pyrantel pamoate and piperazine phosphate in the treatment of Ascaris. Amer. J. Trop. Med. Hyg., 20, 4 : 584-588.

6. Bradley (R. E.) and Conway (D. P.), 1970. - Evaluation of pyrantel Hydrochloride as an anthelmintic in dogs. Vet Med. Small Animal Clinician, 65, $\mathrm{n}^{\circ}$ 8: 767-769.

7. Bumbalo (T. S.), Fugazzotto (D. J.) and Wyczalek (J. V.), 1969. - Treatment of enterobiasis with pyrantel pamoate. Amer. J. Trop. Med. 18 (1) : 50-52.

8. Burriel (L. M.), Fernandey Aguado (P.), Gomez Hernandez (O.) and Bachiller (L.). - Ensayo clinico preliminar de un nuevo farmaco (pyrantel) en el tratamiento del parasitisme per oyures. Med. Klin., 86, 63-67.

9. Cornwell (R. L.) and Jones (R. M.), 1968. - Anthelmintic activity of pyrantel pamoate against Ancylostoma caninum in dogs. J. Trop. Med. Hyg., 71, 165-166.

10. Desowitz (R.S.), Bell (T.), Williams (J.), Cardines (R.) and Tamarua Manca, 1970. - Anti-helmintitic activity of pyrantel pamoate. Am. J. Trop. Med. and Hyg., 19, $\mathrm{n}^{\circ} 5,775-778$.

11. Dessors (J.-P.), 1971. - Efficacité du tartrate de Pyrantel dans le traitement des nématoses intestinales du Chien. Thèse Doctorat Vétérinaire. Faculté de Médecine de Toulouse.

12. EuZÉBY (J.), 1958. - Diagnostic expérimental des helminthoses animales. Vigot Edit., Paris. 
13. EuzéBy (J.), 1963. Les maladies vermineuses des animaux domestiques et leurs incidences sur la pathologie humaine. T. $1 \mathrm{vol}$. 2, Vigot Edit., Paris.

14. Gibson (T. E.), 1965. - Veterinary anthelmintic medication 2nd edition C.A.B. Technical communication, $n^{\circ} 33$.

15. Guarnicara (D.), Léonardi (G.), Ricci (P.) and Ceccarelli (G.), 1986. - Il pamoato di pirantel nella terapia delle infestazioni de E. vermicularis. Pediatria Internazionale XVII, 255.

16. Howes (H. L.) and LyNCH (J. E.), 1967. - Anthelmintic studies with Pyrantel ; its therapeutic and prophylactic efficacy against the enteral stages of various helminths in mice and dogs. J. Parasitology, 53 (5), 1085-1091.

17. Коваулаsi (A), 1970. - Anthelmintic effect of Pyrantel pamoate (Combantrin) against ascariasis, 2nd Int. Cong. Parasitology Proceed. J. Parasitology, 57, 19 Sect. II, Part. $1, \mathrm{n}^{\circ} 900$.

18. Magaudda (P. L.), 1970. - Pyrantel pamoate in the therapy of Enterobius vermicularis infestation. 2nd Int. Cong. Parasitology Proceed. J. Parasitology, 57, 27, 28, Sect. II, Part. 1, $\mathrm{n}^{\circ} 912$.

19. Miller (T. A.), 1971. - Vaccination against the canine hookworm diseases, pp. 153183, in Advances in Parasitology Ben Dawes Editor Academic Press Publisher.

20. Plessis (G.), 1971. - Epidemiologie et prophylaxie de l'ancyclostomose canine. Observations sur une meute du Haut-Anjou. Essais de prévention par le Pamoate de Pyrantel. Thèse Doctorat Vétérinaire. Faculté de Médecine de Créteil.

21. Raynaud (J.-P.), 1970. - Etude de l'efficacité d'une technique de coproscopie quantitative pour le diagnostic de routine et le contrôle des infestations parasitaires des bovins, ovins, équins et porcins. Annales de Parasitologie, t. 45, (3), 1970, 321-342.

22. Taffs (L. F.), Sellers (K. C.), Clark (C. J.) and Froy (D. G.), 1969. - The interpretation of Esophagostomum spp. egg counts in growing pigs: correlation between egg counts and worm burdens. Vet. Record 85, 614-616. 